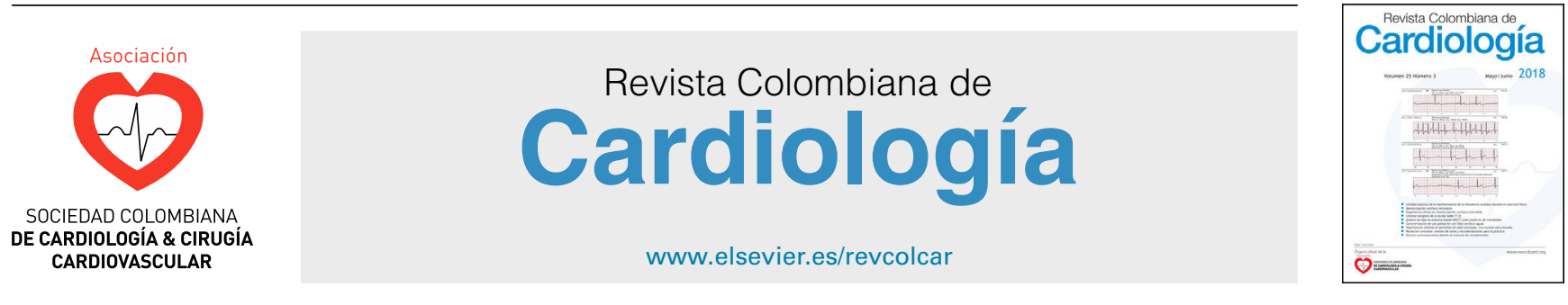

CARDIOLOGÍA DEL ADULTO - REVISIÓN DE TEMAS

\title{
Abordaje de las cardiopatías familiares desde la Medicina genómica
}

\author{
Juan Pablo Trujillo-Quintero a,b,*,1, Julián Palomino-Doza ${ }^{c, 1}$, \\ Ivonne Cárdenas-Reyes ${ }^{\mathrm{a}, \mathrm{b}}$, Juan Pablo Ochoa ${ }^{\mathrm{a}, \mathrm{b}}$ y Lorenzo Monserrat ${ }^{\mathrm{a}, \mathrm{b}}$
}

\author{
a Instituto de investigación Biomédica de A Coruña. INIBIC, A Coruña, España \\ b Departamento clínico Health in Code, A Coruña, España \\ c Unidad de cardiopatías familiares. Servicio de Cardiología. Hospital Universitario 12 de Octubre, Madrid, España
}

Recibido el 14 de julio de 2017; aceptado el 18 de enero de 2018

Disponible en Internet el 20 de junio de 2018

\author{
PALABRAS CLAVE \\ Miocardiopatías; \\ Muerte súbita \\ cardiaca; \\ Asociación \\ genotipo-fenotipo; \\ Secuenciación masiva
}

\section{KEYWORDS}

Cardiomyopathies;

Sudden cardiac

death;

Genotype-phenotype association;

Massive sequencing

\begin{abstract}
Resumen Las cardiopatías familiares son un grupo de enfermedades con alta heterogeneidad clínica y genética. Debido a que pueden heredarse y a su asociación con la muerte súbita, se recomienda efectuar un estudio clínico y genético del individuo afectado y su familia a través de una unidad especializada. Con la implementación de la secuenciación masiva se ha facilitado el acceso a los estudios genéticos en la práctica clínica de forma más rutinaria. Sin embargo, dada la gran cantidad de información obtenida se hacen necesarios el análisis y la interpretación adecuada de los resultados para garantizar un diagnóstico correcto. Este nuevo modelo de medicina amplía nuestra comprensión sobre estas patologías, gracias a que optimiza el diagnóstico, da una mejor aproximación pronóstica de los pacientes e identifica individuos asintomáticos en riesgo. Este artículo pretende realizar una revisión de la arquitectura genética de las enfermedades cardíacas hereditarias y proporcionar un enfoque práctico acerca de la utilidad de la Medicina genómica en el diagnóstico, la estratificación del riesgo y el estudio familiar en pacientes con este tipo de patologías.

(c) 2018 Sociedad Colombiana de Cardiología y Cirugía Cardiovascular. Publicado por Elsevier España, S.L.U. Este es un artículo Open Access bajo la licencia CC BY-NC-ND (http:// creativecommons.org/licenses/by-nc-nd/4.0/).
\end{abstract}

\section{Approach to familial heart diseases from Genomic Medicine}

Abstract The familial heart diseases are a group of diseases with high clinical and genomic heterogeneity. As they can be inherited and are associated with sudden death, it is recommended to perform a clinical and genetic study of the individual affected, as well as the family, in a specialised unit. The implementation of massive sequencing has meant that access to genetic studies is available in the most routine clinical practice. However, due to the large amount of

\footnotetext{
* Autor para correspondencia.

Correos electrónicos: jpatruj@hotmail.com, pablo.trujillo@healthincode.com (J.P. Trujillo-Quintero).

1 Los autores han contribuido en igual medida en este trabajo.
} 
information obtained, the results have to analysed and interpreted to ensure a correct diagnosis. This new medicine model widens the understanding of these diseases, as due to the diagnosis being optimised, it provides a more accurate prognosis for the patients, and identifies asymptomatic individuals at risk. A review is presented on the genetic architecture of heritable heart disease and provides a practical approach on the usefulness of Genomic Medicine in the diagnosis, risk stratification, and the familial study in patients with these types of heart diseases.

(c) 2018 Sociedad Colombiana de Cardiología y Cirugía Cardiovascular. Published by Elsevier España, S.L.U. This is an open access article under the CC BY-NC-ND license (http:// creativecommons.org/licenses/by-nc-nd/4.0/).

\section{Introducción}

Las cardiopatías familiares hacen referencia a aquellas enfermedades cardiovasculares de causa genética, y que en consecuencia, pueden tener una presentación familiar. Entre estas se incluyen miocardiopatías, canalopatías, enfermedades de dilatación aórtica y otros síndromes hereditarios que afectan al sistema cardiovascular ${ }^{1,2}$. Aunque tienen alta variabilidad clínica, comparten como característica en común su asociación con muerte súbita, que en algunos casos puede ser la primera o única manifestación clínica ${ }^{2}$. Por estas razones, es importante establecer programas enfocados hacia la valoración clínica y genética tanto del paciente como de sus familiares, con el fin de identificar portadores asintomáticos que se puedan beneficiar de medidas preventivas para eventos cardiovasculares. Dada la alta heterogeneidad genética de estas enfermedades, múltiples variantes en distintos genes pueden generar fenotipos clínicos similares, por tanto es necesario llevar a cabo un estudio genético completo que permita identificar los defectos genéticos causales ${ }^{1}$. Con la implementación de las plataformas comerciales de secuenciación masiva o Next Generation Sequencing, hoy en día es posible llevar a cabo dicho estudio mediante un único test ${ }^{1,2}$. Aunque estas nuevas plataformas hacen posible secuenciar el genoma de un individuo a partir de una cantidad mínima de ADN extraído de sangre o saliva, solo un adecuado análisis e interpretación permitirán llegar a un diagnóstico preciso.

Este artículo pretende realizar una revisión de la arquitectura genómica de las cardiopatías heredables y proporcionar un enfoque práctico acerca de la utilidad de las tecnologías de secuenciación y de la gestión de la información como herramientas fundamentales en el diagnóstico y la mejor aproximación pronóstica de estas patologías. Este nuevo enfoque de la medicina busca identificar y comprender la predisposición/resistencia genética del individuo para desarrollar enfermedades e implica un nuevo modelo de medicina: predictiva, de precisión y personalizada.

\section{Gestión adecuada de la información en la era genómica}

La secuenciación masiva permite analizar gran cantidad de $A D N$ a un costo cada vez más razonable, convirtiéndose en una herramienta clínica altamente costo-eficiente para el estudio de estas enfermedades. Esta tecnología está permitiendo la identificación de cientos de variantes patogénicas y asociaciones causales con el desarrollo de enfermedad, ampliando nuestro conocimiento acerca de las correlaciones genotipo-fenotipo y explorando mecanismos alternativos de patogenicidad $^{3,4}$.

Estas plataformas de secuenciación requieren una serie de procesos comunes: fragmentación del ADN, purificación, enriquecimiento, obtención de librerías, secuenciación y análisis bioinformático ${ }^{3}$ (fig. 1). La información obtenida, limitada por la complejidad de la arquitectura genética y la gran cantidad de datos generados, debe ser almacenada de la mejor manera para el análisis adecuado y la gestión e interpretación precisa. Por ello se requiere mayor cantidad de recursos humanos y tecnológicos con el fin de garantizar la calidad de los resultados. Otra consecuencia de este aumento de datos es que cada vez es mayor el número de variantes de significado clínico incierto detectadas en cada estudio ${ }^{4}$. Aún no se dispone de un conocimiento suficiente para entender por completo la arquitectura genética de las cardiopatías heredables y de sus variantes asociadas.

Finalmente, la secuenciación masiva puede estar dirigida a una porción del genoma, como es el caso de exoma, o a un conjunto predefinido de genes de interés como serían los paneles específicos de genes. Dada la sensibilidad de los secuenciadores de nueva generación, cada fragmento debe ser leído varias veces y tener cobertura óptima para garantizar la calidad y la fiabilidad de la secuencia analizada. En este sentido, la secuenciación de paneles de genes dirigidos ofrece una mejor cobertura de las regiones de interés que la secuenciación de porciones más grandes del genoma, aspecto crucial para garantizar un análisis completo y adecuado. A su vez, cuanto mayor número de regiones analizadas, mayor número de datos que requieren ser almacenados y analizados adecuadamente; aumentando la posibilidad de identificar variantes de significado incierto o variantes clínicamente relevantes relacionadas con enfermedades que no eran objeto de este estudio (hallazgos incidentales).

Por todo lo anterior es necesario disponer de una plataforma tecnológica de almacenamiento de datos y de un equipo multidisciplinario especializado que realice el análisis de los mismos integrando la información básica y clínica, 


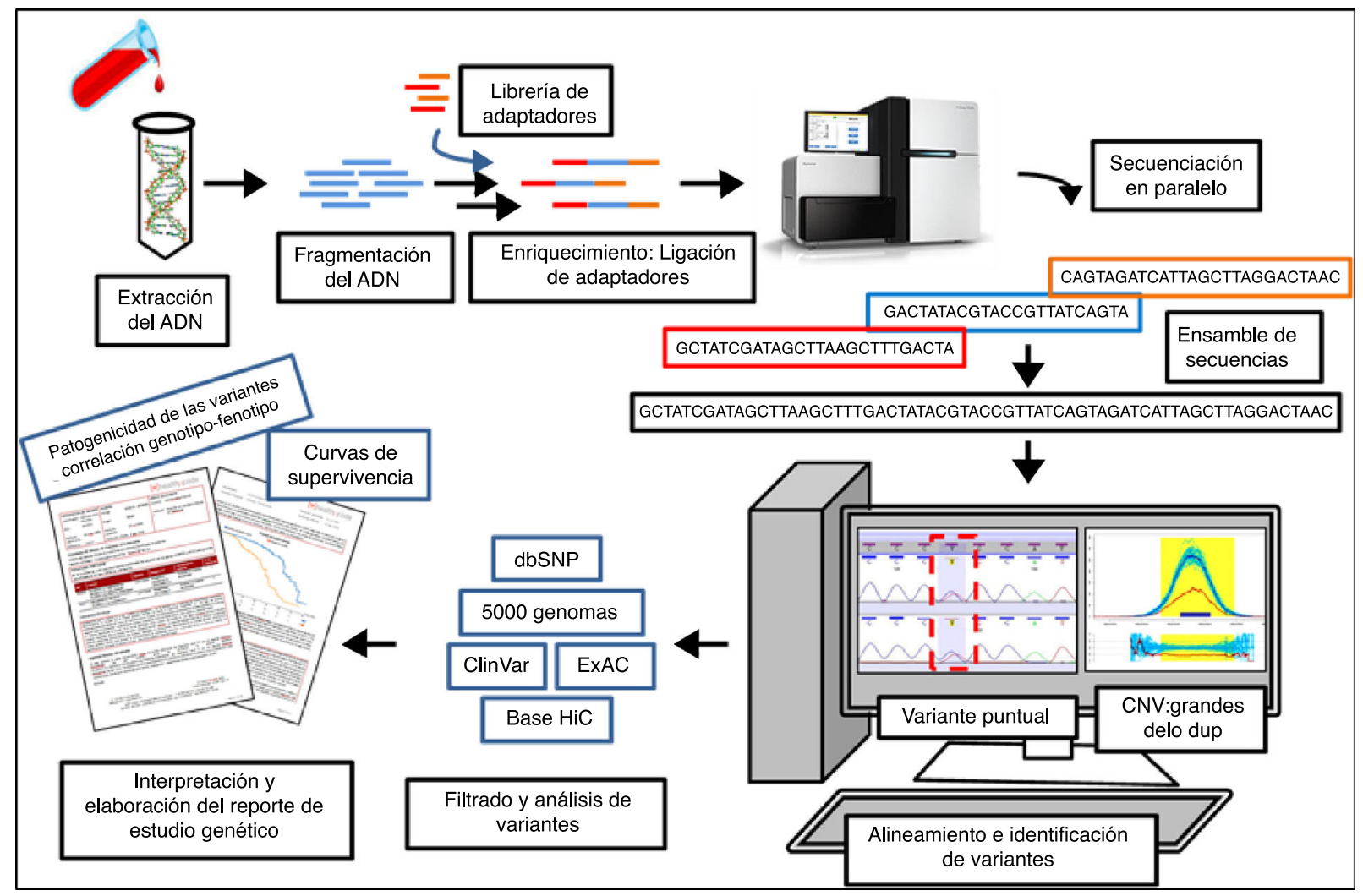

Figura 1 Proceso de secuenciación del ADN mediante secuenciación masiva (NGS) y gestión de la información. De una muestra de ADN se puede secuenciar parte o todo el genoma en un mismo ensayo. Este tipo de estudio genera una gran cantidad de información que requiere un análisis exhaustivo por un equipo multidisciplinar con el fin de lograr una adecuada interpretación de los datos. Este último proceso es importante para la elaboración de informes genéticos que sean de utilidad en la práctica clínica.

enfocando su tarea a identificar asociaciones genotipofenotipo con un alto nivel de evidencia clínica.

\section{Genética clínica de las cardiopatías familiares}

Las cardiopatías familiares pueden presentar diferentes patrones de herencia: autosómicas dominantes o recesivas, ligadas al $\mathrm{X}$ y de patrón matrilineal o mitocondrial. Este aspecto puede ayudar a orientar el diagnóstico, pero dado que alteraciones en múltiples genes se pueden asociar al desarrollo de una misma enfermedad, su diagnóstico clínico correcto no siempre es fácil ${ }^{2,5}$. En algunos casos la presencia de genotipos complejos, de acuerdo con el número de genes implicados o de factores ambientales como el deporte competitivo o la edad, influyen en la expresión de la enfermedad ${ }^{5}$. En este panorama, genes diferentes pueden dar lugar a una expresión clínica similar (un fenotipo similar), pero también un mismo gen puede causar varios fenotipos distintos ${ }^{6}$. Incluso una misma mutación puede ser causa de una expresividad variable del fenotipo ${ }^{7,8}$ (fig. 2B). A toda esta variabilidad contribuyen factores genéticos, epigenéticos y ambientales adicionales parcialmente comprendidos y no siempre identificables (fig. 2A).

Por otra parte, no siempre todos los individuos portadores de variantes patogénicas desarrollarán enfermedad, y en ocasiones la aparición de una sola variante puede no ser causa suficiente para su expresión.

\section{¿Por qué hacer un estudio genético y cómo realizar la valoración clínica-genética en la familia?}

Existen recomendaciones específicas para la realización de un estudio genético en cada grupo de estas enfermedades que se revisarán más adelante. Sin embargo, las indicaciones generales podrán resumirse en las siguientes:

1. Para realizar un diagnóstico etiológico adecuado ${ }^{9,10}$. La identificación causal de la enfermedad es indispensable para el establecimiento de una terapia médica propicia y una mejor intervención preventiva de eventos cardiovasculares.

2. Para facilitar el screening familiar. En el caso de enfermedades heredables los familiares deben someterse a evaluaciones periódicas con la consiguiente carga asistencial y psicológica que esto conlleva. Conocer la variante o variantes patogénicas de la enfermedad en el caso índice de la familia permite identificar a los portadores, y tranquilizar a aquellos que no lo son, finalizando su seguimiento ${ }^{9,10}$.

3. Como herramienta de mejor aproximación pronóstica. Las cardiopatías familiares son clínica y genéticamente heterogéneas. La estratificación pronóstica es una parte esencial de la evaluación de este grupo de pacientes. 

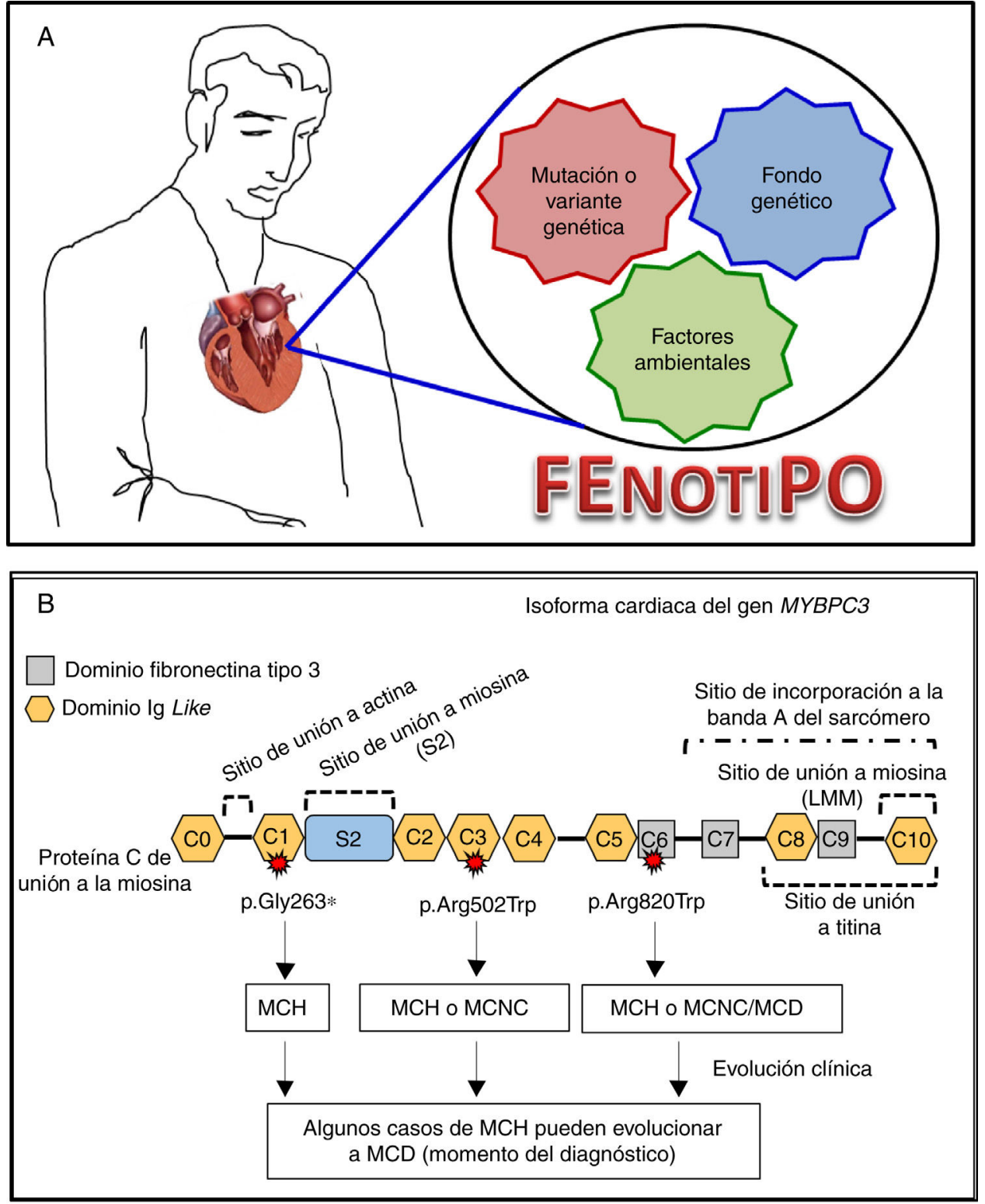

Figura 2 Correlación clínico-molecular de variantes patogénicas en las miocardiopatías. A. La expresión clínica de la enfermedad es el resultado de la interacción de una mutación con factores genéticos (presencia de segundas mutaciones en el mismo u otros genes) y/o ambientales (deporte, miocarditis, entre otros) adicionales. B. Diferentes variantes patogénicas en un gen sarcomérico (se pone de ejemplo al gen MYBPC3 que codifica la isoforma cardiaca de proteína $\mathrm{C}$ de unión a la miosina) pueden asociarse a diferentes tipos de miocardiopatías. A su vez, una misma variante puede dar lugar a diferentes fenotipos, incluso entre portadores de una misma familia. MCH: miocardiopatía hipertrófica; MCD: miocardiopatía dilatada; MCNC: miocardiopatía no compactada.

El conocimiento de una variante patogénica permite realizar un pronóstico asociado a ésta, constituyéndose en un elemento adicional en la estratificación del riesgo, complementando los estudios clínicos tradicionales (fig. 3) ${ }^{11}$.

4. Para dar consejo genético. El asesoramiento genético comprende el proceso de explicar las consecuencias y naturaleza de la enfermedad, las probabilidades de desarrollarla y transmitirla, y las opciones de tratamiento y planificación familiar para las parejas con deseo de futura descendencia $^{12}$.
5. Como herramienta de investigación clínica. Estas enfermedades aún están parcialmente comprendidas, por lo cual no se dispone de información clínica suficiente, estratificación pronóstica, y en muchos casos, ni de terapias médicas adecuadas. El mejor entendimiento de la etiología, el curso natural y el pronóstico de cada una de estas patologías permitirá avanzar en estos aspectos.

6. Para identificar casos beneficiarios de un tratamiento específico. Existe un subgrupo de enfermedades asociadas con el desarrollo de la miocardiopatía hipertrófica, 


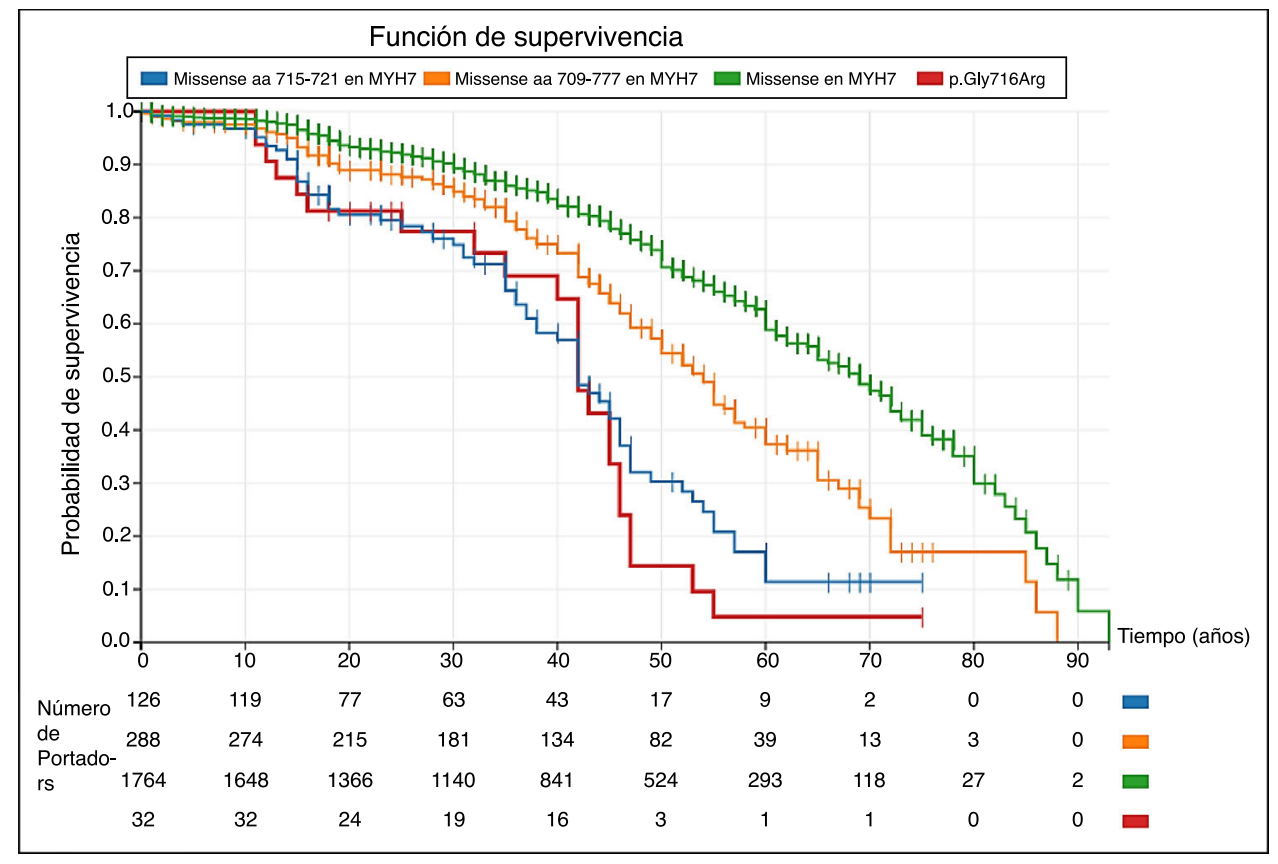

Figura 3 Curvas de sobrevida en portadores de variantes tipo missense en el gen MYH7 (Kaplan-Meier). En las curvas se observa una mayor incidencia de eventos cardiovasculares en los portadores de las variantes de tipo missense localizadas entre los aminoácidos 715 a 721 (incluyendo la variante p.Gly716Arg) al compararlas con las variantes del mismo tipo en el resto del gen 9 . Este gráfico permite comprender varios aspectos relevantes: primero, no todas variantes del mismo tipo descritas en un gen no tienen por qué tener un comportamiento clínico similar; segundo, el pronóstico de los portadores puede variar según la localización de la variante dentro del gen; y tercero, la caracterización clínica de los portadores permite identificar regiones críticas del gen que ayudan a establecer una mejor valoración del pronóstico en sus portadores. Los eventos cardiovasculares fueron definidos como: muerte súbita cardiaca, muerte por insuficiencia cardiaca o trasplante, muerte por accidente cerebrovascular, relacionada con intervención cardiovascular o por otras causas cardiovasculares, descarga apropiada del desfibrilador.

como es la enfermedad de Fabry, que en la actualidad cuenta con un tratamiento específico ${ }^{13}$. El diagnóstico de estas entidades en la mayoría de los casos es de tipo molecular, en cuyo caso el estudio genético permite hacer un diagnóstico diferencial temprano.

La valoración clínica debe ser el primer paso antes de realizar un estudio genético en una familia. Los protocolos de las unidades de cardiopatías familiares sugieren una valoración cardiológica completa, incluyendo ECG y ecocardiograma, principalmente en los miembros de primer grado del individuo afectado y hasta donde sea posible ${ }^{2}$. La historia clínica debe incluir una buena anamnesis, antecedentes personales y familiares, número de afectados y grado de parentesco, hallazgos positivos en el examen físico e identificación de otras alteraciones físicas que pueden ser cruciales para orientar el diagnóstico. En este punto, realizar en la consulta un árbol genealógico permitirá detallar de forma fácil y sencilla los datos familiares clínicamente relevantes. Estos árboles familiares deben incluir datos de al menos tres a cuatro generaciones, ampliando el árbol en la rama familiar, materna o paterna, donde se identifiquen otros miembros afectados. El estudio genético inicialmente solo está indicado en el caso índice de la familia (individuo afectado), previo consentimiento informado. Siempre debe explicarse al paciente sobre sus beneficios, consecuencias y limitaciones. Los resultados, tanto positivos como negativos, deben ser explicados por un especialista experto en estas patologías. Una vez identificados los miembros en riesgo y la causa genética en el caso índice, se debe intentar evaluar de manera organizada a los familiares del caso índice para hacer el estudio de cribado genético o de cosegregación de la variante con la enfermedad $^{2,9}$.

\section{¿Cómo realizar la valoración de la patogenicidad de una variante genética y cómo interpretar el resultado de un estudio genético?}

La interpretación de los resultados de un estudio genético tiene grandes repercusiones en la valoración clínicagenética tanto del individuo afectado como de su familia. La asignación cuidadosa de la patogenicidad de una variante es un aspecto clínicamente relevante en los reportes de los resultados de un estudio genético (tabla 1$)^{14}$. Para la valoración de la patogenicidad de una variante genética se deben tener en cuenta varios aspectos: si la variante se encuentra reportada en la literatura asociada a enfermedad, si está demostrada su cosegregación con una enfermedad específica, su frecuencia alélica en la población general, el tipo de variante, su localización a nivel del gen/proteína, el grado de conservación del aminoácido afectado y su impacto sobre las propiedades fisicoquímicas de la proteína 
Tabla 1 Clasificación de las variantes de acuerdo con la información disponible

\begin{tabular}{ll}
\hline Clasificación de la variante & Utilidad clínica \\
\hline Patogénica & Valor clínico predictivo \\
Criterios mayores & de enfermedad \\
•Variantes previamente descritas asociadas a enfermedad con un alto nivel de evidencia clínica & Para consejo genético \\
y consenso acerca de su patogenicidad. & Recomendado estudio \\
- Cosegregación demostrada con un fenotipo específico: identificación en al menos cinco & familiar \\
probandos (casos índice) o cosegregación en al menos dos familias distintas. & Diagnóstico prenatal \\
-Variante de tipo truncamiento (tipo frameshift, nonsense o que afecta a los sitios canónicos & o preimplantacional
\end{tabular}

de splicing $[+1,+2,-1,-2]$ ) en un gen donde la pérdida de función ha sido demostrada

claramente como mecanismo de patogenicidad y que cumpla al menos un criterio de apoyo.

Criterios de apoyo

a. Variante de tipo truncamiento (tipo frameshift, nonsense o que afecta al splicing) en un gen donde la pérdida de función es relevante.

b. Presentación de novo (padres no afectos y no portadores de la variante).

c. Estudios funcionales que sugieren un efecto deletéreo o no tolerable.

d. Variante missense que genera el mismo cambio de aminoácido que una variante previamente descrita como patogénica.

e. No identificada en población control o con muy baja frecuencia alélica a nivel poblacional $(\mathrm{MAF}<0,001 \%)$

\section{Muy posiblemente patogénica}

\section{Criterios mayores}

-Variante de tipo truncamiento en un gen donde la pérdida de función es relevante y que cumpla al menos un criterio de apoyo.

-Variante missense o deleción in frame (que no altera el marco de lectura) en zona no repetitiva del gen con correlación genotipo-demostrada y que cumpla al menos 2 criterios de apoyo.

\section{Criterios de apoyo}

a. Variante localizada en una región donde se han descrito variantes asociadas con enfermedad.

b. Presentación de novo

c. Cosegregación con la enfermedad o presente en varios casos índices con el mismo fenotipo clínico, pero sin cumplir criterios para ser patogénica.

d. Estudios funcionales que sugieren que podrían ser patogénica.

e. No identificada en población control o con muy baja frecuencia alélica $(M A F<0,001 \%)$

Posiblemente patogénica

Criterios mayores

-Variante de tipo truncamiento en un gen donde la pérdida de función no se ha comprobado como mecanismo patogénico o no cumple criterios para considerarla patogénica.

-Variante intrónica fuera de los sitios relevantes para el proceso de corte y empalme del ARN (splicing) pero cuyo análisis bioinformático sugiere que afectaría este proceso.

-Variante missense o deleción in frame en zona no repetitiva del gen que no cumple criterios para ser patogénica pero que cumple con tres criterios de apoyo.

Criterios de apoyo

a. Identificada en población control con muy baja frecuencia alélica $(M A F<0,01 \%)$.

b. Presentación de novo asociada a un nuevo fenotipo clínico en la familia.

c. Estudio clínico y genético del paciente y familiares sugieren una correlación de la variante con la enfermedad.

d. Localizada en un dominio o región relevante del gen en el que previamente se han descrito variantes patogénicas.

e. Descrita en al menos dos individuos no relacionados con el mismo fenotipo clínico.

f. Estudios bioinformáticos coinciden en que tendría un efecto deletéreo.

Valor clínico predictivo de enfermedad Consejo genético Recomendado estudio familiar

Por el momento sin valor clínico predictivo de enfermedad La evaluación de cosegregación puede ser útil para definir su patogenicidad 
Tabla 1 (continuación)

\begin{tabular}{|c|c|}
\hline Clasificación de la variante & Utilidad clínica \\
\hline $\begin{array}{l}\text { Patogenicidad incierta o de significado clínico incierto } \\
\text { Criterios } \\
\text {-Variantes con información contradictoria respecto a su patogenicidad (no se ha confirmado } \\
\text { cosegregación con la enfermedad; no hay estudios funcionales o estos no son concluyentes) } \\
\text {-No cumplen criterios para ser incluidas en otra categoría de la clasificación. }\end{array}$ & $\begin{array}{l}\text { Por el momento sin valor } \\
\text { clínico predictivo de } \\
\text { enfermedad } \\
\text { Evaluación de } \\
\text { cosegregación si hay } \\
\text { familiares afectados y } \\
\text { sólo en el contexto de } \\
\text { investigación }\end{array}$ \\
\hline $\begin{array}{l}\text { Posiblemente no patogénica o benigna } \\
\text { Criterios mayores } \\
\text { - La frecuencia alélica de la variante en población control es mayor que la esperada para la } \\
\text { enfermedad, o presenta una alta frecuencia alélica poblacional (>0,05\% pero } \leq 0,5 \%) \text {. } \\
\text {-Está descrita en individuos con fenotipos no relacionados. } \\
\text {-No cosegregación con la enfermedad en al menos un familiar. }\end{array}$ & $\begin{array}{l}\text { Sin valor clínico } \\
\text { predictivo de } \\
\text { enfermedad } \\
\text { No recomendado } \\
\text { incluirlas en el estudio } \\
\text { genético familiar }\end{array}$ \\
\hline
\end{tabular}

- Cumple al menos dos criterios de apoyo.

Criterios de apoyo

Variante de tipo missense en un gen en donde sólo las variantes de tipo truncamiento han demostrado ser causa de enfermedad.

Estudio funcional que demuestre que la variante no altera la estructura o función de la proteína que codifica.

Predictores bioinformáticos coincidentes en que la variante no alteraría la función de la proteína (se incluyen las variantes intrónicas fuera de la zona relevante de splicing). Inserciones/deleciones in frame en una región repetitiva del gen sin función conocida. Presencia de la variante en homocigosis en población control.

No patogénica o benigna

Criterios mayores

-Identificada en población control con una frecuencia alélica muy alta (similar a la de un polimorfismo $>0,5-1 \%$ ) o con alta evidencia clínica acerca de su no patogenicidad.

-No presenta cosegregación con la enfermedad en al menos dos familias.

- Cumple al menos cuatro de los criterios de apoyo.

Criterios de apoyo

Frecuencia alélica mayor que la esperada para la enfermedad, o MAF $>0,05 \%$.

Ausencia de cosegregación de la variante con el fenotipo clínico en al menos un familiar.

Estudio funcional que demuestre que la variante no altera la estructura o la función de la proteína que codifica.

Presencia de la variante en individuos sanos (no afectados) a una edad en la cual la enfermedad debería haberse expresado.

Modificada de Standards and guidelines for the interpretation of sequence variants: a joint consensus recommendation of the American College of Medical Genetics and Genomics and the Association for Molecular Pathology.

y los resultados de estudios funcionales existentes y/o de los predictores bioinformáticos ${ }^{1,14}$. Es importante recalcar que la mayoría de estas variables son solo datos orientativos a tener en cuenta dentro de esta valoración y que el aspecto más importante como evidencia de patogenicidad es la confirmación de su cosegregación con la enfermedad.

Cuanto más rara es una variante genética, menor es su frecuencia alélica en la población general y mayor es la probabilidad de que pueda asociarse a enfermedad. De la misma manera, es poco probable que las variantes genéticas consideradas polimorfismos (frecuencias alélicas en población general $>0,5 \%$ ) sean causales de enfermedad en forma aislada (fig. 4). En general, se considera que las variantes ausentes o con frecuencias muy bajas en población general, aquellas reportadas previamente en la literatura asociadas con el desarrollo de enfermedad, o que afectan regiones funcionalmente relevantes a nivel de proteína, tienen mayor probabilidad de ser patogénicas. En la tabla 1 se presenta una clasificación de las diferentes categorías en las que se puede enmarcar un hallazgo de un estudio genético, en tanto que en la figura 4 se muestra una correlación entre la frecuencia poblacional de una variante y su posible patogenicidad.

Por consenso, si hay dudas en la patogenicidad de la variante genética, "de significado clínico incierto", no se recomienda su utilización con valor clínico predictivo de enfermedad $^{1,9,14}$. Es decir, que hasta no tener más información no debería ser utilizada para la toma de decisiones clínicas en sus portadores. En algunos casos seleccionados es recomendable realizar el cribado familiar y evaluar la cosegregación de la variante con el fenotipo clínico, y determinar si solo está presente en individuos afectados o también en sanos, en un contexto de investigación. Si estas 


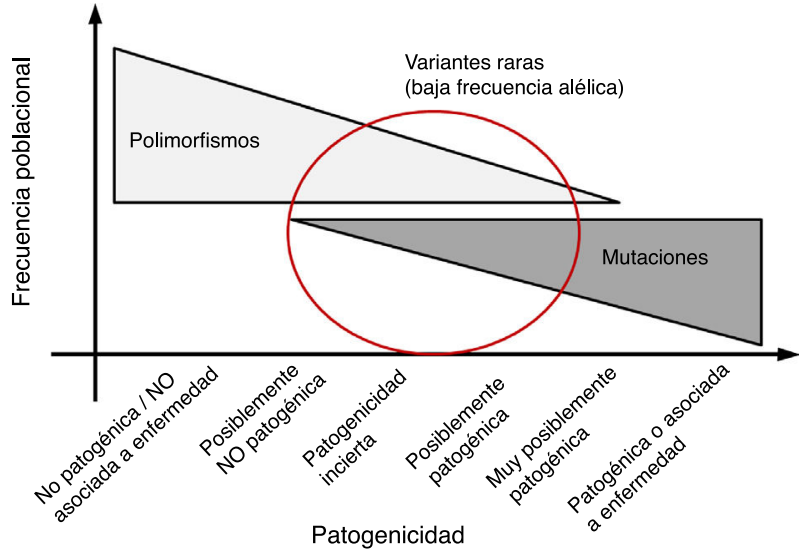

Figura 4 Relación entre la frecuencia de una variante y su posible patogenicidad. En general las variantes con alta frecuencia alélica poblacional (polimorfismo) tienen baja probabilidad de ser patogénicas; mientras que variantes no presentes en la población general tienen alta probabilidad de ser patogénicas. Sin embargo, en la parte media del espectro existe un solapamiento entre variantes raras (con baja frecuencia alélica) que pueden ser causa de enfermedad o por el contrario no ser patogénicas.

variantes son reclasificadas como patogénicas, pueden ser utilizadas con fines clínicos predictivos de enfermedad. Es importante remarcar que ser portador de una variante patogénica no implica estar enfermo, sino presentar una mayor predisposición que la población general a desarrollar una enfermedad específica, razón por la cual se requiere un seguimiento adecuado.

En general, los estudios genéticos que permiten identificar variantes patogénicas o probablemente patogénicas son considerados resultados informativos, ya que podrían modificar el manejo médico del paciente y sus familiares identificados como portadores. Por el contrario, aquellos estudios que no identifican variantes clínicamente relevantes son considerados como resultados no informativos, ya que no permiten cambiar el manejo médico del paciente, ni identificar familiares en riesgo de desarrollar la enfermedad, y en este caso se considera a todos sus miembros población en riesgo. La identificación de variantes de patogenicidad incierta debe considerarse como un resultado no informativo, con la salvedad de que en aquellos casos en los que se establezca su patogenicidad, se demuestre su cosegregación con la enfermedad o sea posteriormente descrita como claramente asociada a enfermedad, pasaría a ser un resultado informativo.

\section{Cardiopatías familiares}

\section{Miocardiopatías}

La Sociedad Europea de Cardiología define a las miocardiopatías como alteraciones miocárdicas en las cuales el músculo cardiaco está estructural y/o funcionalmente alterado, en ausencia de factores predisponentes, enfermedad coronaria, hipertensión, enfermedad valvular o enfermedad cardiaca congénita, que pueda explicar dichas anomalías $^{15}$. Pertenecen a este grupo las miocardiopatías hipertrófica, dilatada, arritmogénica, restrictiva y la no compactada 9 . En este apartado se comentarán particularmente las tres primeras, por ser las más frecuentes. Para consultar datos acerca de la genética de las otras miocardiopatías se recomienda al lector referirse a los documentos de consenso de las Sociedades Europea y Americana de Cardiología ${ }^{16,17}$.

La miocardiopatía hipertrófica de define como la presencia de hipertrofia ventricular izquierda en ausencia de factores hemodinámicos que la expliquen ${ }^{15,17}$. Es una enfermedad genética, con un patrón de herencia autosómico dominante. Se estima que la prevalencia de esta enfermedad es de 1:500 adultos ${ }^{17,18}$. De acuerdo con datos norteamericanos, la miocardiopatía hipertrófica es responsable de la mayor parte de las muertes súbitas que se producen en sujetos jóvenes, antes de 35 años de edad, aparentemente sanos, y particularmente en la subpoblación de atletas. La incidencia anual de muerte súbita en pacientes con miocardiopatía hipertrófica varía entre 0,1 y 1,7\%; se acepta como regla general que es $<1 \%$, con subgrupos de pacientes de alto riesgo en donde la misma podría ser mayor $^{19}$.

Entre el $50-70 \%$ de los pacientes con miocardiopatía hipertrófica son portadores de al menos una variante patogénica en un gen sarcomérico, siendo algo mayor este porcentaje en las formas que cumplen criterios clínicos y de presentación familiar, y disminuyendo en aquellos que presentan formas esporádicas o cuando el diagnóstico no es claro $^{20,21}$. Los genes más implicados en el desarrollo de miocardiopatía hipertrófica son MYH7 y MYBPC3, que codifican para la cadena pesada de la beta miosina y la proteína $C$ de fijación a la miosina, respectivamente, ya que explican hasta el $50 \%$ de los $\operatorname{casos}^{20,22}$. Desde 1990 se han descrito más de 1.500 mutaciones asociadas al desarrollo de miocardiopatía hipertrófica en múltiples genes, particularmente en genes sarcoméricos. Sin embargo, otros genes no sarcoméricos se han asociado progresivamente con el desarrollo de la enfermedad (tabla 2). También es importante tener en cuenta la presencia de fenocopias de la miocardiopatía hipertrófica (fenotipos clínicos similares), que en su mayoría se trata de enfermedades de depósito, enfermedades de Fabry, Pompe y Danon, déficit de PRKAG2, cuyo diagnóstico diferencial en muchos casos solo es posible mediante el estudio genético.

En las guías de actuación de la Sociedad Europea de Cardiología se recomienda el estudio genético en todos los pacientes con diagnóstico de miocardiopatía hipertrófica y familiares en riesgo (nivel de evidencia lb) ${ }^{23}$.

La miocardiopatía arritmogénica se caracteriza por el reemplazo del tejido miocárdico ventricular por tejido fibroso y adiposo, asociado a un riesgo elevado de arritmias ventriculares. Desde el punto de vista epidemiológico, tiene una prevalencia de entre 1:2.000 a 1:5000 individuos de población general ${ }^{24}$. Su diagnóstico clínico se basa en una serie de criterios arrítmicos, electrocardiográficos, morfológicos y familiares, pero suele ser complejo ya que la expresión de la enfermedad muestra gran variabilidad. Tradicionalmente se acepta que el mecanismo de herencia es autosómico dominante, y en algunos casos la sola presencia de una variante es suficiente para que el fenotipo se exprese; algunas pueden asociarse a un mal pronóstico. Sin embargo, no es infrecuente que una 
Tabla 2 Genes relacionados con el desarrollo de las diferentes miocardiopatías y rendimiento del test genético en estas entidades

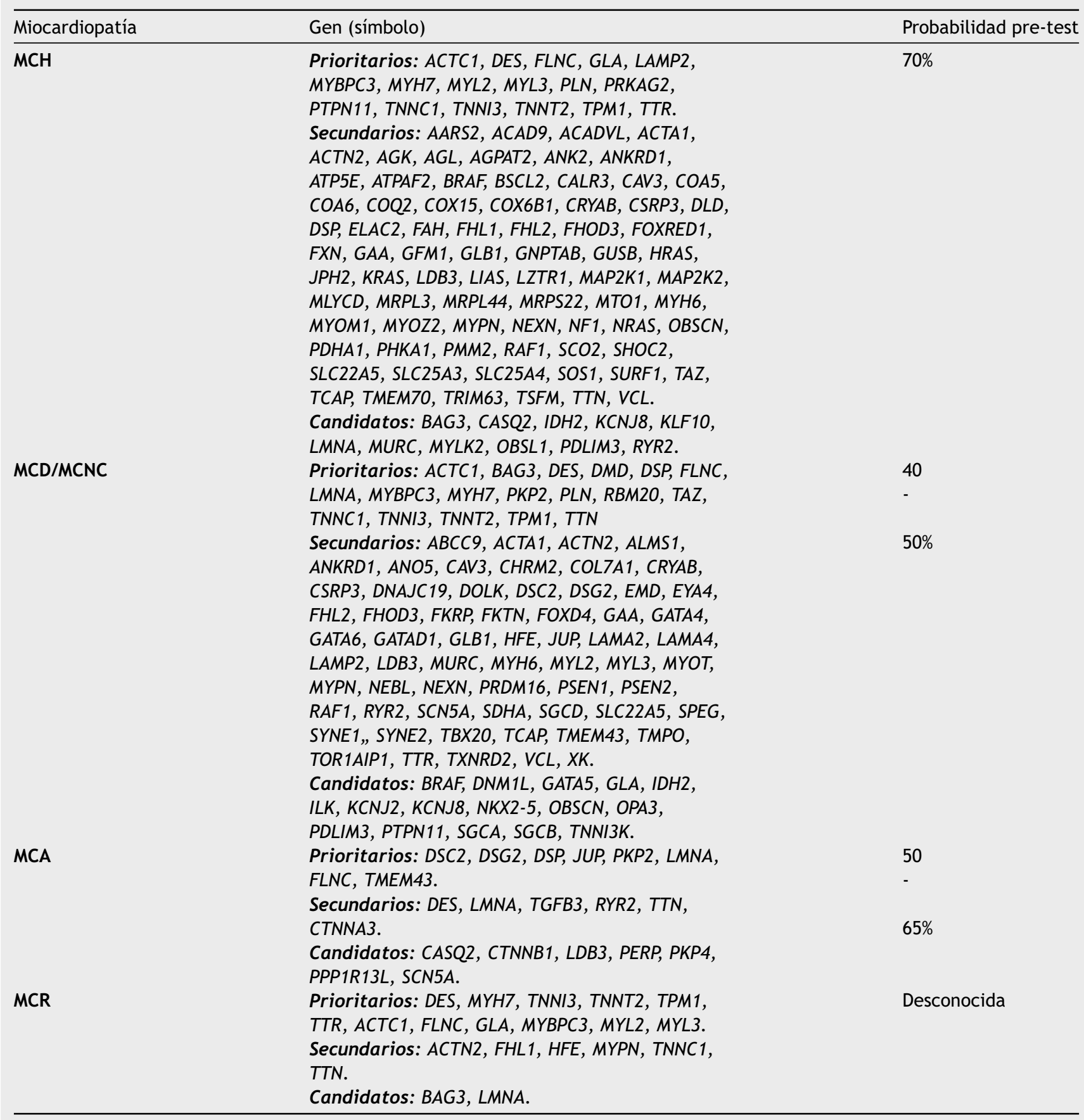

MCH: miocardiopatía hipertrófica; MCD: miocardiopatía dilatada; MCA: miocardiopatía aritmogénica; MCNC: miocardiopatía no compactada, MCR: miocardiopatía restrictiva.

variante genética considerada patogénica requiera de factores adicionales genéticos, presencia de segundas variantes, o ambientales, deporte competitivo, infecciones virales, etc., para producir enfermedad ${ }^{25}$. También existen formas recesivas de miocardiopatía arritmogénica caracterizadas por afectación extracardiaca (de piel y faneras), como es el caso de la enfermedad de Naxos y Carvajal ${ }^{25}$. En general, aproximadamente el $60 \%$ de los pacientes con diagnóstico de miocardiopatía arritmogénica son portadores de una variante patogénica causal identificable ${ }^{26}$.

La miocardiopatía arritmogénica se ha asociado principalmente con mutaciones en los genes desmosomales (DSC2, DSG2, DSP, JUP, PKP2 (tabla 2). El gen comúnmente identificado es el $P K P 2$, seguido de la DSP y DSG2. Como se mencionaba previamente, es frecuente que exista más de una variante genética causal en una familia afectada y que 
esta combinación explique el desarrollo de la enfermedad. Una excepción son las variantes patogénicas en el gen DSP, y en especial las variantes de tipo "radical" que generarían truncamientos a nivel de la proteína, en donde la sola presencia de una variante puede explicar el fenotipo, a veces asociándose a formas predominantemente izquierdas que pueden ser indistinguibles de una miocardiopatía dilatada. A su vez, también existen genes no desmosomales con variantes patogénicas claramente descritas como causa de la enfermedad. Algunos ejemplos son DES, FLNC, PLN y TMEM43.

La miocardiopatía dilatada se define como la presencia la dilatación del ventrículo izquierdo y disfunción sistólica ventricular izquierda en ausencia de condiciones que alteren el llenado, tales como hipertensión, enfermedad valvular o enfermedad arterial coronaria suficiente para causar deterioro de la función sistólica. Se cree que la incidencia de la enfermedad es de 1 en 2.500 individuos de la población general, si bien esta podría ser aún mayor ${ }^{27}$. Aunque durante muchos años se pensó que la causa era exclusivamente autoinmune o viral, hoy se acepta que más de la mitad de los casos tienen una etiología genética, en los cuales se podría identificar una variante causal o patogénica. Gran parte de estos casos entra en la definición de miocardiopatía dilatada familiar, en la que se requiere que más de un miembro de la familia se encuentre afectado $^{28}$. La enfermedad presenta gran morbimortalidad asociada, con alta tasa de muerte súbita asociada a arritmias ventriculares (entre 15 y $50 \%$ a 5 años) ${ }^{29}$. El patrón de herencia más frecuente en esta entidad es autosómico dominante, aunque existen formas ligadas al $X$ y recesivas. Hasta la fecha, las variantes de tipo truncamiento en el gen de la Titina, TTN, representan la causa conocida más frecuente de miocardiopatía dilatada ${ }^{30,31}$. Mutaciones en genes sarcoméricos, filamentos intermedios, proteínas estructurales, factores de transcripción, y proteínas de la membrana nuclear también han sido asociadas al desarrollo de la enfermedad (tabla 2). A medida que aumentan los conocimientos sobre la enfermedad, es mayor la cantidad de genes identificados. Recientemente Ortiz-Genga et al. describieron que las variantes de tipo truncamiento en el gen FLNC asociadas con el desarrollo de un fenotipo solapado de miocardiopatía dilatada/miocardiopatía arritmogénica que se caracteriza por la presencia de arritmias ventriculares frecuentes tienen una alta tasa de muerte súbita en las familias portadoras ${ }^{32}$.

El consenso de expertos de la Sociedad Europea de Cardiología recomienda el estudio genético en todos los pacientes con defectos de conducción o historia familiar de muerte súbita (recomendación clase I) y en pacientes con miocardiopatía dilatada familiar (recomendación Ila) ${ }^{2,26}$.

\section{Canalopatías cardiacas}

Dentro de esta definición se incluye un grupo heterogéneo de enfermedades primarias que producen algún trastorno de la excitabilidad de las membranas de las células cardiacas. Este grupo de patologías están asociadas a eventos de muerte súbita de causa arrítmica. En su mayoría, son el producto de alteraciones en los genes que codifican para canales iónicos del músculo cardíaco, o proteínas que modulan su función. Aunque comparten al igual que otras cardiopatías heredables una gran heterogeneidad clínica y genética, presentan una alta penetrancia incompleta; solo algunos de los portadores de variantes patogénicas expresan la enfermedad. Este último aspecto dificulta muchas veces los estudios genéticos familiares orientados a confirmar su cosegregación con la enfermedad.

El síndrome de QT largo se caracteriza por un alargamiento del intervalo QT en el electrocardiograma de superficie derivado de una prolongación excesiva de la repolarización ventricular, velocidad de las células miocárdicas para volver a su potencial de membrana en reposo. Esto hace que esté asociado a una alta tasa de muerte súbita por arritmias ventriculares con una incidencia anual, que ronda el $0,9 \%^{33}$. La prevalencia del síndrome de QT largo en población general es aproximadamente 1:2.000 individuos ${ }^{34}$.

Desde el punto de vista hereditario existen formas autosómicas dominantes, síndrome de Romano-Ward, y recesivas como el síndrome de Jervell y Lange-Nielsen, que incluye sordera neurosensorial congénita y otras alteraciones extracardiacas y solo es producido por mutaciones en los genes KCNQ1 y KCNE1. Variantes patogénicas en al menos 8 genes han sido asociadas con el desarrollo de síndrome de QT largo (tabla 3). El estudio genético puede identificar una variante causal en aproximadamente $75 \%$ de los casos, siendo los genes más frecuentemente asociados el KCNQ1, KCNH2 y el SCN5A que corresponden al síndrome de QT largo tipo 1, 2 y 3 , respectivamente. Estos tres genes son responsables del $90 \%$ de los casos en los pacientes con genotipo positivo ${ }^{26}$. El consenso de expertos de la Sociedad Europea de Cardiología recomienda al menos el estudio genético para QT largo 1,2 y 3 en todos los pacientes con diagnóstico probable de QT largo, sin causas que lo puedan explicar (recomendación clase (c) $)^{2,26}$.

El síndrome de Brugada es una enfermedad rara caracterizada por anomalías en el electrocardiograma de superficie, elevación con pendiente negativa del ST en precordiales derechas y riesgo elevado de muerte súbita de causa arrítmica. Es responsable del 4 al $12 \%$ de los casos de muerte súbita cardiaca y de hasta el $50 \%$ de la muerte súbita en corazones estructuralmente normales ${ }^{35}$.

Se hereda de forma autosómica dominante con penetrancia incompleta y alta heterogeneidad genética. Aproximadamente el $50 \%$ de los pacientes con este síndrome tienen historia familiar de la enfermedad. Sin embargo, sólo el $30 \%$ son portadores de una variante genética que explique el fenotipo (tabla 3 ). Las variantes patogénicas en SCN5A, subunidad alfa del canal de sodio, son responsables de aproximadamente el $80 \%$ de los casos en los pacientes con genotipo positivo ${ }^{35}$. El consenso de expertos de la Sociedad Europea de Cardiología recomienda el estudio genético en pacientes con diagnóstico de Brugada tipo 1 (recomendación clase Ila) y lo desaconseja en aquellos con patrón 2 o 3 aislado $^{2,26}$.

\section{Enfermedades hereditarias de la aorta}

Este grupo de enfermedades incluye algunos trastornos hereditarios del tejido conectivo que pueden llevar a dilatación/aneurisma y/o ruptura/disección de la aorta. 
Tabla 3 Genes relacionados con el desarrollo de las diferentes canalopatías y rendimiento del test genético en estas entidades

\begin{tabular}{|c|c|c|}
\hline Canalopatía & Gen (símbolo) & Probabilidad pre-test \\
\hline SQTL & $\begin{array}{l}\text { Prioritarios: KCNQ1 (tipo 1), KCNH2 (tipo 2), } \\
\text { SCN5A (tipo 3), KCNJ2 (tipo 7), KCNE1 (tipo 5), } \\
\text { KCNE2 (tipo 6), CACNA1C (tipo 8). } \\
\text { Secundarios: ANK2 (tipo 4), CAV3, SCN4B, AKAP9, } \\
\text { SNTA1, KCNJ5, RYR2, CALM1, CALM2, CALM3, } \\
\text { KCND2, TRDN. } \\
\text { Candidatos: HCN4, KCNA5, KCND3, KCNE3, } \\
\text { KCNE5, NOS1AP, PTRF, SCN1B. }\end{array}$ & $70 \%$ \\
\hline SQTC & $\begin{array}{l}\text { Prioritarios: } K C N H 2, K C N Q 1, K C N J 2 \\
\text { Secundarios: } C A C N A 1 C, C A C N A 2 D 1, C A C N B 2 \text {. } \\
\text { Candidatos: CACNA1D. }\end{array}$ & Desconocido \\
\hline $\mathrm{SBr}$ & $\begin{array}{l}\text { Prioritarios: SCN5A, CACNA1C, CACNA2D1, } \\
\text { CACNB2, KCNJ8, SCN1B. } \\
\text { Secundarios: SCN10A, ABCC9, ANK2, GPD1L, } \\
\text { HCN4, KCND2, KCND3, KCNE3, KCNE5, PKP2, } \\
\text { RANGRF, SCN2B, SCN3B, SLMAP, TRPM4. } \\
\text { Candidatos: ANK3, CACNA1D, FGF12, KCNH2. }\end{array}$ & $30 \%$ \\
\hline TVPC & $\begin{array}{l}\text { Prioritarios: RYR2, CASQ2, KCNJ2. } \\
\text { Secundarios: CALM1, CALM2, CALM3, TRDN, } \\
\text { ANK2. } \\
\text { Candidatos: SCN } 5 A \text {. }\end{array}$ & $\begin{array}{l}50 \\
- \\
60 \%\end{array}$ \\
\hline
\end{tabular}

SQTL = síndrome de QT largo; SQTC = síndrome de QT corto; SBr = síndrome de Brugada; TVPC = taquicardia ventricular polimórfica catecolaminérgica.

Entre estos trastornos se incluyen los síndromes de Marfan, Loeys-Dietz, Ehlers-Danlos tipo vascular, el de aneurismaosteoartrosis, Shprintzen-Goldberg, el de tortuosidad arterial y el aneurisma o disección de aorta familiar ${ }^{36,37}$. Estos síndromes pueden presentar características clínicas comunes, como dismorfismo facial, anomalías oculares y alteraciones esqueléticas, que dificultan su diagnóstico clínico. Sin embargo, algunas de estas patologías presentan un comportamiento vascular más agresivo que otras, haciéndose fundamental su adecuado diagnóstico diferencial para realizar una mejor aproximación pronóstica. Los pacientes con síndrome de Loeys-Dietz o de síndrome de aneurisma-osteoartrosis pueden presentar afectación en más de una rama arterial, aorta, coronarias, cerebrales, etc., y eventos de disección incluso sin dilatación vascular previa. La implementación de la secuenciación masiva en muchos casos permite aclarar la etiología genética, orientando, confirmando o replanteando el diagnóstico clínico. En este sentido, se convierte en una herramienta clínica de diagnóstico y de mejor aproximación pronóstica, ya que permite la evaluación y el seguimiento clínico adecuados $^{37,38}$.

Respecto al rendimiento del test genético en estas enfermedades, utilizando un diseño adecuado de los genes incluidos en el panel a secuenciar y con una orientación clínica apropiada, la confirmación diagnóstica puede llegar a más del $90 \%$ en el caso del síndrome de Marfan, $70-95 \%$ en el síndrome de Loeys-Dietz y $20 \%$ en el síndrome de aneurisma y/o disección de la aorta torácica familiar ${ }^{9}$. En la actualidad, el estudio genético para los síndromes de Marfan y LoeysDietz está incluido dentro de los criterios diagnósticos ${ }^{2,38}$.
En la tabla 4 se muestran los genes más comúnmente asociados con cada una de estas enfermedades y el rendimiento de la secuenciación masiva en estas entidades.

\section{Conclusiones}

Las nuevas tecnologías de secuenciación masiva han revolucionado el estudio de las causas genéticas de la enfermedad en la práctica clínica. La secuenciación masiva permite obtener gran cantidad de información en un periodo corto de tiempo y a un precio razonable, pero requiere la implementación de plataformas bioinformáticas específicas y la conformación de equipos especializados en esta tarea, así como una adecuada interpretación y gestión del conocimiento.

Las cardiopatías heredables son un grupo de enfermedades caracterizadas por una alta heterogeneidad clínica y genética. Todas ellas tienen en común asociarse con riesgo de muerte súbita, muchas veces como única manifestación de la enfermedad.

El estudio genético en cardiopatías heredables permite estudiar la etiología de la enfermedad, dar una mejor aproximación pronóstica, plantear tratamientos más precisos en algunos casos, optimizar el screening familiar, dar consejo genético y permitir a la familia tener una descendencia libre de enfermedad.

La implementación de las nuevas tecnologías de secuenciación junto con una adecuada gestión de la información e interpretación de los resultados constituyen uno de los 
Tabla 4 Genes relacionados con el desarrollo de las diferentes patologías de dilatación/disección aórtica y/o síndromes vasculares de causa genética y rendimiento del test genético en estas entidades

\begin{tabular}{|c|c|c|}
\hline $\begin{array}{l}\text { Enfermedades de la aorta de } \\
\text { causa genética }\end{array}$ & Gen (símbolo) & Probabilidad pre-test \\
\hline Síndrome de Marfan & FBN1 & $\sim 70-90 \%$ \\
\hline Síndrome de Loeys-Dietz & $\begin{array}{l}\text { TGFBR2, TGFBR1, TGFB2, } \\
\text { TGFB3 }\end{array}$ & $\sim 70-90 \%$ \\
\hline $\begin{array}{l}\text { Síndrome de } \\
\text { aneurisma-osteoartrosis }\end{array}$ & SMAD3 & Desconocido \\
\hline $\begin{array}{l}\text { Síndrome de tortuosidad } \\
\text { arterial }\end{array}$ & SLC2A10 & Desconocido \\
\hline Síndrome de Ehlers-Danlos & $\begin{array}{l}\text { COL3A1 (tipo vascular), } \\
\text { COL1A1, COL1A2, COL5A1, } \\
\text { COL5A2, ADAMTS2, B4GALT7, } \\
\text { CHST14, FKBP14, PLOD1, } \\
\text { SLC39A13 }\end{array}$ & Depende del fenotipo sospechado \\
\hline Síndrome de Beals & FBN2 & $\sim 40-70 \%$ \\
\hline $\begin{array}{l}\text { Síndrome de } \\
\text { Shprintzen-Goldberg }\end{array}$ & SKI & Desconocido \\
\hline Síndrome de Williams & $E L N$ & Desconocido \\
\hline Síndrome de Costello & HRAS & $\sim 80-90 \%$ \\
\hline $\begin{array}{l}\text { Aneurisma y/o disección de } \\
\text { aorta torácica familiar }\end{array}$ & $\begin{array}{l}\text { ACTA2, TGFBR2, TGFBR1, } \\
\text { MYH11, SMAD3, SMAD4, MYLK, } \\
\text { FBN1, MFAP5, MAT2A, PRKG1, } \\
\text { LOX, FOXE3. }\end{array}$ & $\sim 15-20 \%$ \\
\hline $\begin{array}{l}\text { Heterotopia nodular } \\
\text { periventricular }\end{array}$ & FLNA & $\sim 90 \%$ \\
\hline $\begin{array}{l}\text { Síndromes de diagnóstico } \\
\text { diferencial y otras colagenosis } \\
\text { (sin dilatación aórtica) }\end{array}$ & $\begin{array}{l}\text { ATP7A, ADAMTSL4, B3GAT3, } \\
\text { EFEMP2, MED12, NOTCH1, } \\
\text { ZDHHC9, etc. }\end{array}$ & - \\
\hline $\begin{array}{l}\text { Homocisteinuria (diagnóstico } \\
\text { diferencial) }\end{array}$ & $\begin{array}{l}\text { CBS, MTHFR, MTR, MTRR, } \\
\text { MMADHC }\end{array}$ & $\sim 90 \%$ \\
\hline
\end{tabular}

ejemplos evidentes del nuevo modelo de medicina: predictiva, de precisión y personalizada.

\section{Financiación}

Ninguna.

\section{Conflicto de intereses}

Cuatro de los autores pertenecen al Departamento clínico Health in Code, empresa de diagnóstico genético de enfermedades cardiovasculares.

\section{Agradecimientos}

A todos los clínicos que durante años han venido depositando su confianza en nuestro centro con el fin de construir nuevo conocimiento en cardiopatías familiares.

A nuestro personal de laboratorio y bioinformático que son parte fundamental de este equipo de trabajo.

A nuestro grupo de traducción por su colaboración en los apartados en inglés de este artículo.

\section{Bibliografía}

1. Montserrat L, Mazzanti A, Ortiz-Genga M, Barriales-Villa R, Garcia-Giustiniani D, Gimeno-Blanes JR. The interpretation of genetic tests in inherited cardiovascular diseases. Cardiogenetics. 2011;1:e8.

2. Barriales-Villa R, Gimeno-Blanes JR, Zorio-Grima E, Ripoll-Vera T, Evangelista-Masip A, Moya-Mitjans A, et al. Plan of Action for Inherited Cardiovascular Diseases: Synthesis of Recommendations and Action Algorithms. Rev Esp Cardiol (English ed). 2016;69:300-9.

3. Ware JS, Roberts AM, Cook SA. Next generation sequencing for clinical diagnostics and personalised medicine: implications for the next generation cardiologist. Heart. 2012;98:276-81.

4. Teekakirikul P, Kelly MA, Rehm HL, Lakdawala NK, Funke BH. Inherited cardiomyopathies: molecular genetics and clinical genetic testing in the postgenomic era. JMD. 2013;15:158-70.

5. Colhoun HM, McKeigue PM, Davey Smith G. Problems of reporting genetic associations with complex outcomes. Lancet. 2003;361:865-72.

6. Monserrat L, Hermida-Prieto M, Fernandez X, Rodríguez I, Dumont C, Cazón L, et al. Mutation in the alpha-cardiac actin gene associated with apical hypertrophic cardiomyopathy, left ventricular non-compaction, and septal defects. Eur Heart J. 2007;28:1953-61. 
7. Bezzina C, Veldkamp MW, van Den Berg MP, Postma AV, Rook $\mathrm{MB}$, Viersma JW, et al. A single $\mathrm{Na}(+)$ channel mutation causing both long-QT and Brugada syndromes. Circulation Research. 1999;85:1206-13.

8. Bezzina C, Veldkamp MW, van Den Berg MP, Postma AV, Rook MB, Viersma JW, et al. Genetics of cardiomyopathies: novel perspectives with next generation sequencing. Current pharmaceutical design. 2015;21:418-30.

9. Cirino AL, Harris S, Lakdawala NK, Michels M, Olivotto I, Day SM, et al. Role of genetic testing in inherited cardiovascular disease. JAMA Cardiol. 2017;2:1153-60.

10. McNally EM. Incorporating genetic testing into cardiovascular practice. JAMA Cardiol. 2017;2:1151-2.

11. Garcia-Giustiniani D, Arad M, Ortiz-Genga M, Barriales-Villa R, Fernández X, Rodríguez-García I, et al. Phenotype and prognostic correlations of the converter region mutations affecting the beta myosin heavy chain. Heart. 2015;101:1047-53.

12. National Society of Genetic Counselors' Definition Task Force, Resta R, Biesecker BB, Bennett RL, Blum S, Hahn SE, et al. A new definition of Genetic Counseling: National Society of Genetic Counselors' Task Force report. J Genet Counsel. 2006;15:77-83.

13. Seydelmann N, Wanner C, Stork S, Ertl G, Weidemann F. Fabry disease and the heart. Best practice \& research Clinical endocrinology \& metabolism. 2015;29:195-204.

14. Richards S, Aziz N, Bale S, Bick D, Das S, Gastier-Foster J, et al. Standards and guidelines for the interpretation of sequence variants: a joint consensus recommendation of the American College of Medical Genetics and Genomics and the Association for Molecular Pathology. Genetics in Medicine: official journal of the American College of Medical Genetics. 2015;17:405-24.

15. Elliott P, Andersson B, Arbustini E, Bilinska Z, Cecchi F, Charron $P$, et al. Classification of the cardiomyopathies: a position statement from the European Society Of Cardiology Working Group on Myocardial and Pericardial Diseases. Eur Heart J. 2008;29:270-6.

16. Charron P, Arad M, Arbustini E, Basso C, Bilinska Z, Elliott P, et al. Genetic counselling and testing in cardiomyopathies: a position statement of the European Society of Cardiology Working Group on Myocardial and Pericardial Diseases. Eur Heart J. 2010;31:2715-26.

17. Richardson P, McKenna W, Bristow M, Maisch B, Mautner B, O'Connell J, et al. Report of the 1995 World Health Organization/International Society and Federation of Cardiology Task Force on the Definition and Classification of cardiomyopathies. Circulation. 1996;93:841-2.

18. Maron BJ, Gardin JM, Flack JM, Gidding SS, Kurosaki TT, Bild DE. Prevalence of hypertrophic cardiomyopathy in a general population of young adults. Echocardiographic analysis of 4111 subjects in the CARDIA Study. Coronary Artery Risk Development in (Young) Adults. Circulation. 1995;92:785-9.

19. Elliott P, McKenna WJ. Hypertrophic cardiomyopathy. Lancet. 2004;363:1881-91.

20. Konno T, Chang S, Seidman JG, Seidman CE. Genetics of hypertrophic cardiomyopathy. Curr Op Cardiol. 2010;25:205-9.

21. Morita H, Rehm HL, Menesses A, McDonough B, Roberts AE, Kucherlapati R, et al. Shared genetic causes of cardiac hypertrophy in children and adults. N Engl J Med. 2008;358:1899-908.

22. Watkins H, Conner D, Thierfelder L, Jarcho JA, MacRae C, McKenna WJ, et al. Mutations in the cardiac myosin binding
protein-C gene on chromosome 11 cause familial hypertrophic cardiomyopathy. Nat Genet. 1995;11:434-7.

23. Elliott PM, Anastasakis A, Borger MA, Borggrefe M, Cecchi F, Charron P, et al. 2014 ESC Guidelines on diagnosis and management of hypertrophic cardiomyopathy: the Task Force for the Diagnosis and Management of Hypertrophic Cardiomyopathy of the European Society of Cardiology (ESC). Eur Heart J. 2014;35:2733-79.

24. Basso C, Corrado D, Marcus FI, Nava A, Thiene G. Arrhythmogenic right ventricular cardiomyopathy. Lancet. 2009;373:1289-300.

25. Nakajima T, Kaneko Y, Irie T, Takahashi R, Kato T, lijima T, et al. Compound and digenic heterozygosity in desmosome genes as a cause of arrhythmogenic right ventricular cardiomyopathy in Japanese patients. Circ J. 2012;76:737-43.

26. Ackerman MJ, Priori SG, Willems S, Berul C, Brugada R, Calkins $\mathrm{H}$, et al. HRS/EHRA expert consensus statement on the state of genetic testing for the channelopathies and cardiomyopathies: this document was developed as a partnership between the Heart Rhythm Society (HRS) and the European Heart Rhythm Association (EHRA). Europace. 2011;13:1077-109.

27. Codd MB, Sugrue DD, Gersh BJ, Melton LJ. Epidemiology of idiopathic dilated and hypertrophic cardiomyopathy. A population-based study in Olmsted County, Minnesota, 19751984. Circulation. 1989;80:564-72.

28. Hershberger RE, Siegfried JD. Clinical and genetic issues in familial dilated cardiomyopathy. J Am Coll Cardiol. 2011;57: 1641-9.

29. Komajda M, Jais JP, Reeves F, Goldfarb B, Bouhour JB, Juillieres $\mathrm{Y}$, et al. Factors predicting mortality in idiopathic dilated cardiomyopathy. Eur Heart J. 1990;11:824-31.

30. Michels VV, Moll PP, Miller FA, et al. The frequency of familial dilated cardiomyopathy in a series of patients with idiopathic dilated cardiomyopathy. New Eng J Med. 1992;326:77-82.

31. Michels VV, Moll PP, Miller FA, Tajik AJ, Chu JS, Driscoll DJ, et al. Truncations of titin causing dilated cardiomyopathy. New Eng J Med. 2012; 366:619-28.

32. Ortiz-Genga MF, Cuenca S, Dal Ferro M, Zorio E, Salgado-Aranda R, Climent V, et al. Truncating FLNC Mutations Are Associated With High-Risk Dilated and Arrhythmogenic Cardiomyopathies. J Am Coll Cardiol. 2016;68:2440-51.

33. Moss AJ, Schwartz PJ, Crampton RS, et al. The long QT syndrome. Prospective longitudinal study of 328 families. Circulation. 1991;84:1136-44.

34. Moss AJ, Schwartz PJ, Crampton RS, Tzivoni D, Locati EH, MacCluer J, et al. Prevalence of the congenital long-QT syndrome. Circulation. 2009;120:1761-7.

35. Brugada P, Brugada J, Roy D. Brugada syndrome 1992-2012: 20 years of scientific excitement, and more. Eur Heart J. 2013;34:3610-5.

36. Morisaki T, Morisaki H. Genetics of hereditary large vessel diseases. J Hum Genet. 2016;61:21-6.

37. Verstraeten A, Luyckx I, Loeys B. Aetiology and management of hereditary aortopathy. Nat Rev Cardiol. 2017; 14:197-208.

38. Loeys BL, Dietz HC, Braverman AC, Callewaert BL, De Backer J, Devereux RB, et al. The revised Ghent nosology for the Marfan syndrome. J Med Genet. 2010;47:476-85. 